\title{
USING PLANAR LASER-INDUCED FLUORESCENCE TO STUDY THE PHASE TRANSFORMATIONS OF TWO-COMPONENT LIQUID AND SUSPENSION DROPLETS
}

\author{
Roman S. Volkov, Geniy V. Kuznetsov, Maxim V. Piskunov, $\mathcal{E}$ \\ Pavel A. Strizhak*
}

\author{
National Research Tomsk Polytechnic University, 30 Lenin Avenue, Tomsk 634050, Russia \\ *Address all correspondence to: Pavel A. Strizhak, National Research Tomsk Polytechnic University, 30 \\ Lenin Avenue, Tomsk 634050, Russia, E-mail: pavelspa@tpu.ru
}

Original Manuscript Submitted: 3/31/2019; Final Draft Received: 4/1/2019

\begin{abstract}
Using the planar laser-induced fluorescence (PLIF), we performed experiments to determine evaporation dynamics of homogeneous and heterogeneous droplets of liquids, conditions of their boiling, and explosive breakup. For the 1-2 $\mathrm{mm}$ water droplets, the distribution of highly non-homogeneous and non-steady temperature field was detected by highspeed cross-correlation video recording and the Tema Automotive software. We identified highly nonlinear dependences of evaporation rate on heating temperature and time as well as water droplet size. For the two-component liquids and water-based suspensions of graphite, we revealed unsteady temperature fields and established mechanisms and regimes of the explosive breakup of the heterogeneous droplets when heated. The regimes differ in the number and dimensions of the emerging gas-liquid fragments as well as the durations of the main stages. The three regimes of warming-up and evaporation of the heterogeneous droplets have been obtained. The explosive breakup of droplets enables provision for the secondary atomization of the liquid with the emergence of an aerosol cloud. The surface area of the liquid increases several-fold. The temperature variations at the water/solid or water/flammable component interfaces were determined corresponding to each boiling and breakup regime. Using the PLIF, we studied reasons and mechanism of the explosive breakup of water droplets with single large carbonaceous inclusions when heated.
\end{abstract}

KEY WORDS: heat transfer enhancement, evaporation, boiling, planar laser-induced fluorescence, temperature field, fluids

\section{INTRODUCTION}

There are a large number of technologies based on the high-temperature evaporation and boiling of liquids, emulsions, and suspensions (Bochkareva et al., 2018; Morozov et al., 2018). These technologies deal with technical limitations of the corresponding units, devices, and productions. Namely, it is difficult to enhance (Sazhin, 2017) the evaporation at the modern industrial plant equipment. It would be very useful to establish the necessary and sufficient conditions of enhanced vaporization. These conditions are critical in such technologies as thermal and flame water purification (Vysokomornaya et al., 2018), syngas production (Zaitsev et al., 2018a), composite fuel production (Glushkov et al., 2016), etc. The critical conditions considerably differ during heating and evaporation of droplets in gaseous media or at the walls of heat chambers. This is due to both the differences between the surface area of energy supply and the different mechanisms of heat transfer, i.e., convective, conductive, radiative, and combined ones. Each of these mechanisms differs in the critical conditions of the intensive vaporization of liquid droplets. The composition of the droplets strongly affects these conditions and the corresponding heat fluxes.

The rapid evaporation and boiling of two-component liquids, emulsions, and solutions are of interest, since a group of components vaporizes simultaneously (Sazhin, 2017). Many high-temperature applications listed above 


\section{NOMENCLATURE}
$R_{d} \quad$ initial radius of two-component liquid droplet $(\mathrm{mm})$
$t$ time (s)
$T$ temperature $\left({ }^{\circ} \mathrm{C}\right)$
$T_{a} \quad$ airflow temperature $\left({ }^{\circ} \mathrm{C}\right)$
$T_{d}$ temperature of the water at the inner interface in a suspension droplet $\left({ }^{\circ} \mathrm{C}\right)$
$T_{s} \quad$ temperature of the free surface of a suspension droplet $\left({ }^{\circ} \mathrm{C}\right)$
$T_{c} \quad$ temperature at the center of the water film (at the bottom of a suspension droplet) enveloping a solid inclusion

or water temperature inside of the two-component liquid droplet $\left({ }^{\circ} \mathrm{C}\right)$

$T_{b} \quad$ temperature at the water-flammable liquid interface $\left({ }^{\circ} \mathrm{C}\right)$

$U_{a} \quad$ air velocity $(\mathrm{m} / \mathrm{s})$

$V_{d} \quad$ water volume $(\mu \mathrm{l})$

$V_{i n c} \quad$ inclusion volume $\left(\mathrm{mm}^{3}\right)$

$y \quad$ length of the droplet section ( $\mathrm{mm}$ )

$\delta \quad$ liquid film thickness around the solid particle (mm)

$\gamma \quad$ volume concentration of the flammable liquids in the two-component droplets (vol. \%)

cannot obtain a sustainable development due to a limited experimental database (Gatapova et al., 2018; Zaitsev et al., 2018b). It is necessary to apply adequate registration methods for the main parameters characterizing the hightemperature processes of such complex liquids. A study of vaporization of two-component liquids is the most suitable, because such an approach will allow focusing on the main physical patterns in detail. It is of great interest to record non-stationary temperature fields during the intensive heating succeeded by the boiling and explosive breakup of two-component liquid droplets (Volkov et al., 2014; Kuznetsov et al., 2016; Nebuchinov et al., 2017).

Study (Nebuchinov et al., 2017) considers a hypothesis that water may overheat significantly, reaching the values above the water-boiling temperature in the presence of solid particles in heterogeneous droplets. These conditions enable observing the considerable droplet surface deformation followed by their disintegration to produce a cloud of much smaller droplets than the initial ones. Unfortunately, the confirmation of this hypothesis is difficult (Volkov et al., 2014). However, the hypothesis of possible explosive breakup of a heterogeneous water droplet when moving through high-temperature gases (Volkov et al., 2014) was confirmed in Kuznetsov et al. (2016). Solids with a high infrared absorption coefficient allow the faster heat-up of water. Then, vapor bubbles develop and fill the heterogeneous droplet. Because of higher vapor pressure inside the droplet as compared to the surface tension pressure, the heterogeneous droplet disintegrates and forms a large group of smaller droplets. The evaporation surface area of water increases several-fold (Kuznetsov et al., 2016). The duration of this process is about 1-3 s. The video frames of the process (Kuznetsov et al., 2016) remind one of droplet explosion. To explain the main mechanism of the explosive breakup of heterogeneous droplets, there is a need for measuring a temperature inside liquid phase of droplets. The temperature of an evaporating heterogeneous droplet of water, only liquid phase, can be measured in any point using contactless methods.

Recent years have seen the development of optical methods for high-speed measurement of the key parameters of gaseous, fluid, and heterogeneous media, such as temperature, speed, consumption, particle size, concentration, etc. The contactless method planar laser-induced fluorescence (PLIF) (Nebuchinov et al., 2017; Strizhak et al., 2017; Volkov and Strizhak, 2017; Piskunov and Strizhak, 2018) is the method of choice to study non-stationary temperature fields of evaporating heterogeneous (including multi-component) droplets. Experimental results (Nebuchinov et al., 2017; Strizhak et al., 2017; Volkov and Strizhak, 2017; Piskunov and Strizhak, 2018) with the multicomponent droplets are discussed in point of the transition time of the temperature field of such droplets from the non-uniform one to the quasi-steady one. This time can be from 5 to $20 \%$ of a lifetime of droplets, i.e., of their complete evaporation time. The lower the heating temperature, the longer the quasi-steady temperature field forms. The higher the concentration of a combustible component, the faster droplets warm up to the conditions when the temperature field is be considered to be quasi-steady, i.e., a temperature differential does not exceed $10^{\circ} \mathrm{C}$. 
Using PLIF will allow us to measure the temperature gradients and temperature variations at the phase interface and boundary between liquid components, necessary for the breakup of heterogeneous droplets and enhancement of their evaporation. The reliable experimental data on the temperature fields of droplets are important for several reasons. First, many water-based compositions have high specific heat and evaporation heat. The analysis of the temperature fields of such droplets enables one to determine how much the heat energy is consumed during the droplet warming-up and endothermic phase transitions. If one had such data, it could be possible to optimize the warming-up and the cooling of droplets. Second, various criteria (Qubeissi et al., 2017; Zubkov et al., 2017; Qubeissi and Sazhin, 2017) are employed when developing the breakup models of the heterogeneous droplets. The use of every criterion can be substantiated if the temperature fields of droplets will be presented. Third, the values of the temperature in the liquid layers inside a droplet versus time and heating temperature will help to determine the droplet sizes necessary for complete evaporation or intensive ignition depending on applications.

The purpose of this research is to determine, based on experiments, the non-stationary temperature fields of two-component liquid and suspension droplets under high-temperature heating followed by explosive breakup and formation of a cloud of fine aerosol. The paper is organized as follows. The experimental setup and the procedure of the experiment are described in Section 2. The results are discussed in Section 3. Section 4 contains concluding remarks.

\section{EXPERIMENTAL SETUP AND PROCEDURE}

Figure 1 presents a scheme of the experimental setup. The system consists of the following equipment: Leister $\mathrm{CH}$ 6060 air blower (air velocity $0-5 \mathrm{~m} / \mathrm{s}$ ); Leister LE $5000 \mathrm{HT}$ air heater (temperature range $20-800^{\circ} \mathrm{C}$ ); quartz-glass cylinder (inner diameter $0.1 \mathrm{~m}$, wall thickness $0.003 \mathrm{~m}$ ); personal computer with Tema Automotive software to track dynamic objects as well as ActualFlow and PLIF Kit software; ImperX B2020M cross-correlation digital camera (recording frequency $25 \mathrm{fps}$, resolution $2048 \times 2048$ pixels); Nikon macro lens (focal length $200 \mathrm{~mm}$ ); a set of

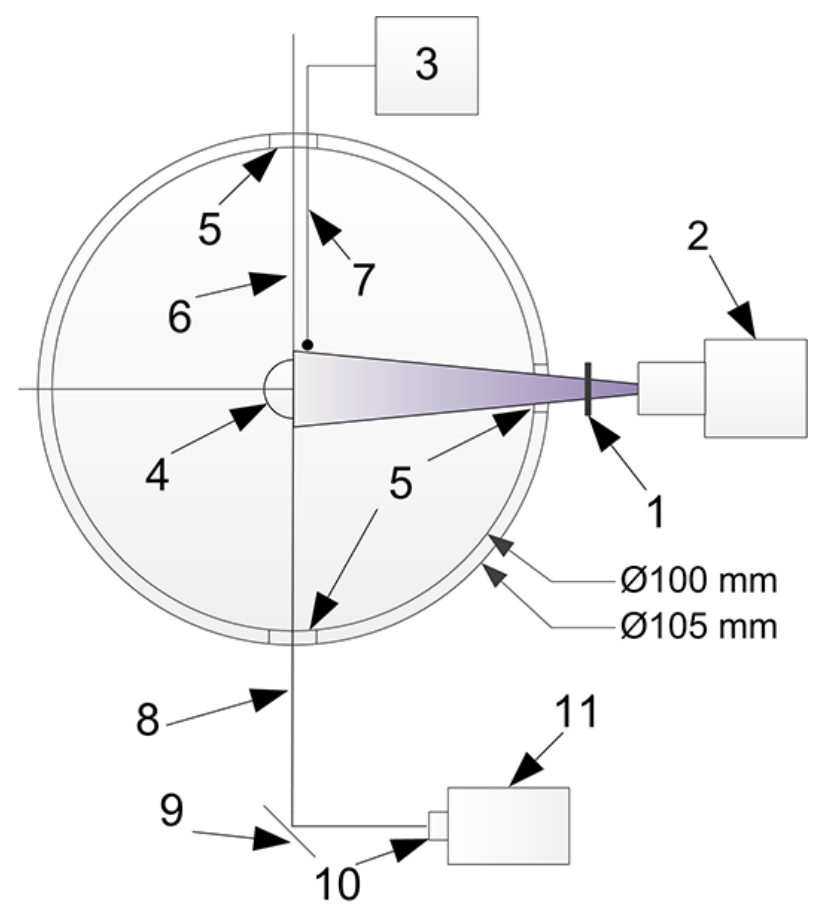

FIG. 1: Schematics of experimental setup and measuring area: (1) light filter; (2) digital cross-correlation video camera equipped with micro lens; (3) data acquisition device; (4) water droplet; (5) $10 \mathrm{~mm}$ hole; (6) droplet holder; (7) fast-response thermocouple; (8) collimated laser light; (9) optical mirror; (10) collimator; (11) Nd:YAG laser 
light filters (600-10 nm) and fluorescent dyes; dual pulsed Nd:YAG Quantel EverGreen 70 laser (wavelength 532 $\mathrm{nm}$, maximum energy $74 \mathrm{~mJ}$ ); a lens for generating a laser sheet with an opening angle of $8^{\circ}$; motorized positioning mechanism; National Instruments 9213 data acquisition module with a $\mathrm{Pt}-(\mathrm{Pt}-\mathrm{Rh})$ fast-response thermocouple (temperature measurement range $0-1600^{\circ} \mathrm{C}$, error $\pm 1{ }^{\circ} \mathrm{C}$, thermal time lag $0.1 \mathrm{~s}$, junction diameter $0.1 \mathrm{~mm}$ ). Hot airflow with controllable parameters, namely temperature $T_{a}$ and air velocity $U_{a}$, is produced by the air blower and air heater. Such a system supplies hot air to the hollow transparent cylinder made of quartz glass. The inner diameter of the cylinder is $0.1 \mathrm{~m}$.

We use distilled water and flammable liquids as the second component of the two-component liquid (Strizhak et al., 2017): oil; kerosene TS-1; gasoline AI-92; fuel oil M-100; waste turbine oil TP-30; and waste transformer oil TSP. The concentration $(\gamma)$ of the flammable liquids in the two-component droplets under study ranges from 10 to 70 vol. $\%$.

The non-transparent graphite inclusions in the shape of a $2 \times 2 \times 3 \mathrm{~mm}$ parallelepiped are applied. A graphite particle is mechanically fixed on a ceramic tip $0.05 \mathrm{~m}$ long.

Distilled water is used for a fluorophore aqueous solution in a mass concentration of $1000 \mu \mathrm{g} / \mathrm{l}$ (Charogiannis et al., 2015) to create a water droplet. Rhodamine B acts as a fluorescence dye. A heterogeneous droplet (Fig. 2) is placed inside the quartz-glass cylinder using the motorized positioning mechanism.

To generate a Rhodamine B/water droplet, we use a single-channel Finnpipette Novus dosing device with a pitch variation of $0.1 \mu \mathrm{l}$. The droplet volume (only Rhodamine B/water) at the beginning of heating is $V_{d}=20 \mu \mathrm{l}$ in the experiments with suspension droplets and $V_{d}=10-25 \mu \mathrm{l}$ in the experiments with two-component droplets. The two-component liquid and suspension droplets are initially generated at room temperature.

Two methods are used for producing a two-component droplet [see Figs. 2(b) and 2(c)]. Note that the position of the inclusion is set with high precision for better operating cross-correlation algorithms. The particle is fixed

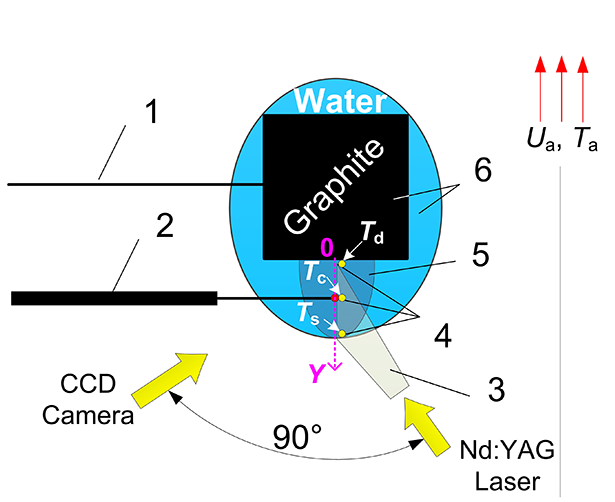

(a)

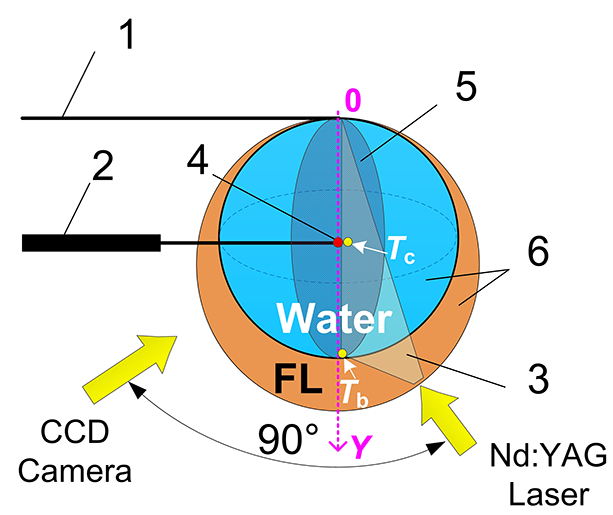

(b)

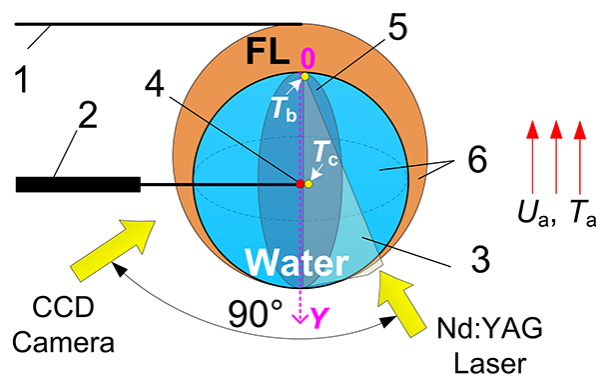

(c)

FIG. 2: Schematics of PLIF measurement during experiments: (1) droplet holder; (2) fast-response thermocouple; (3) light sheet; (4) thermocouple junction; (5) video recording area; (6) heterogeneous droplet; FL - flammable liquid; OY - droplet section 
on the ceramic tip [see Fig. 2(a)] so that the frontal surface of the particle is perpendicular to the video recording direction. The bottom surface of the inclusion looks like a straight line. Figure 2 also illustrates the vertical OY sections and yellow points, in which the temperatures using PLIF were measured as well as the red points, in which the temperatures were determined using a thermocouple.

The PLIF method is based on the natural fluorescence of laser-induced dye molecules. Experiments involve that the droplet is cut with the dual pulsed laser along the symmetry axis with a frequency of $4-10 \mathrm{~Hz}$ depending on the experimental conditions. The light sheet thickness is $100 \mu \mathrm{m}$. The experiments include two stages. The first stage is the calibration of the measurement system, and the second one involves recording the temperature field in the OY section of the heterogeneous droplet. At the first stage, charge-coupled device (CCD) images are produced at temperature constants specified for the entire measuring area. Measurements are provided for at least 20-30 air temperatures $T_{a}$ ranging from 20 to $500^{\circ} \mathrm{C}$. The main objective of these tests is to obtain the calibration curve with high accuracy (Fig. 3) describing the dependence between the temperature $T_{c}$ and Rhodamine B luminosity. To calibrate the PLIF method, we use a miniature thermocouple with a $0.05 \mathrm{~mm}$ diameter. The droplet temperature curve construction was based on the image processing by PLIF Calibration. At the second stage, the heating and evaporation of heterogeneous droplets are recorded with constant parameters of the laser, camera, measurement area, background illumination, and arrangement of devices in the operating area. CCD images are processed with the ActualFlow software. PLIF Reconstruction was used for the recorded results using the calibration curve presented in Fig. 3. To follow the identical initial conditions, 3-5 series of experiments are performed. Each series consists of 100 to 1000 images of the heterogeneous droplet. The error of the droplet temperature $T_{c}$ estimated using our approach (PLIF method) did not exceed $2-3^{\circ} \mathrm{C}$.

\section{RESULTS AND DISCUSSION}

\subsection{Unsteady and Heterogeneous Temperature Fields of Two-Component Liquid and Suspension Droplets}

Figures 4 and 5 present the processed temperature fields and profiles for the droplets under study obtained by PLIF. The result analysis of Figs. 4(a) and 5(b) enables us to draw several conclusions. Temperature fields in droplets remain highly heterogeneous up to $60-70 \%$ of the time necessary for full evaporation.

The temperature variation in the liquid phase of a heterogeneous droplet reaches $30-40^{\circ} \mathrm{C}$. Maximum temperatures $(T)$ are observed near the inclusion/water interface and on the free droplet surface on the side of the incoming hot airflow. An increase in $T$ of the water film near the inclusion/water interface shows the heat accumulation near the solid inclusion surface. This result is also confirmed to compare the complete evaporation time of water in the graphite suspension droplets with the same parameter for the water droplets (Fig. 6). The heat accumulation near the inclusion/water interface drastically affects the complete evaporation time of water. It decreases on average by $39 \%$ at $T_{a}>100^{\circ} \mathrm{C}$ (Fig. 6). The standard deviation of this characteristic is not more than $11 \%$.

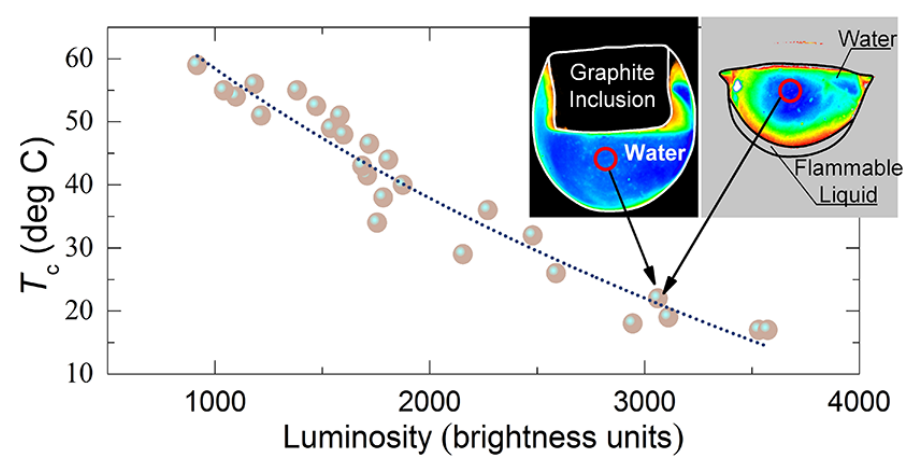

FIG. 3: Experimental calibration curve 


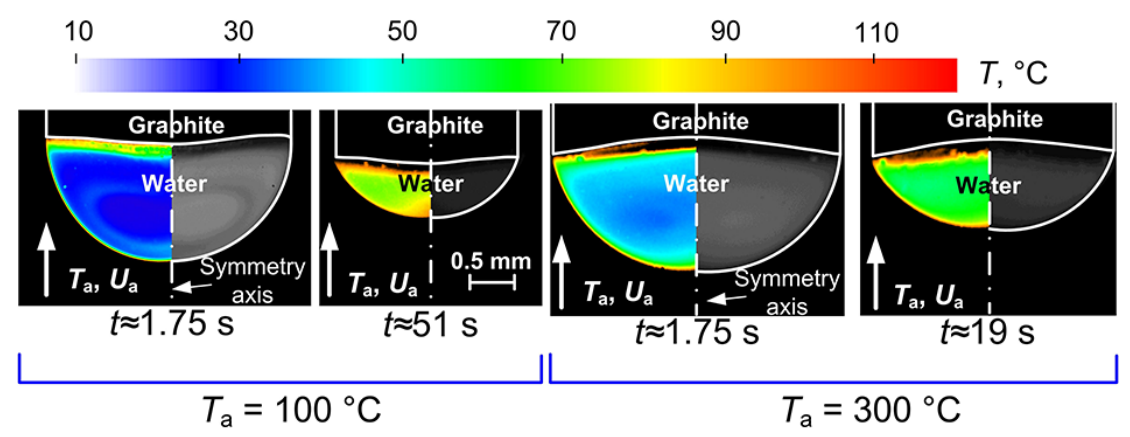

(a)

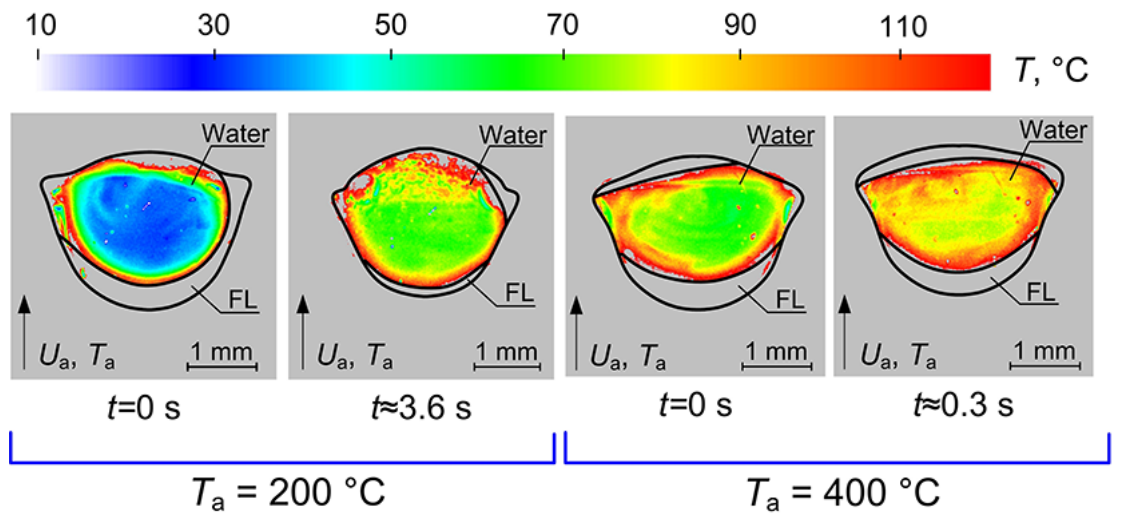

(b)

FIG. 4: Unsteady temperature fields of graphite suspension droplets (initial $V_{d} \approx 20 \mu \mathrm{l}$ and $\delta \approx 2.3 \mathrm{~mm}$ ) at $T_{a} \approx 100^{\circ} \mathrm{C}$ and $T_{a} \approx 300^{\circ} \mathrm{C}$; (right) the image of the droplet obtained from CCD camera; (left) the temperature field in the water layer; unsteady temperature fields of two-component water/kerosene droplet $\left(V_{d} \approx 15 \mu \mathrm{l}, R_{d} \approx 1.53 \mathrm{~mm}, \gamma \approx 50\right.$ vol. $\left.\%, U_{a} \approx 3 \mathrm{~m} / \mathrm{s}\right)$ at $T_{a} \approx 200^{\circ} \mathrm{C}$, and $T_{a} \approx 400^{\circ} \mathrm{C} ; \mathrm{FL}-$ kerosene

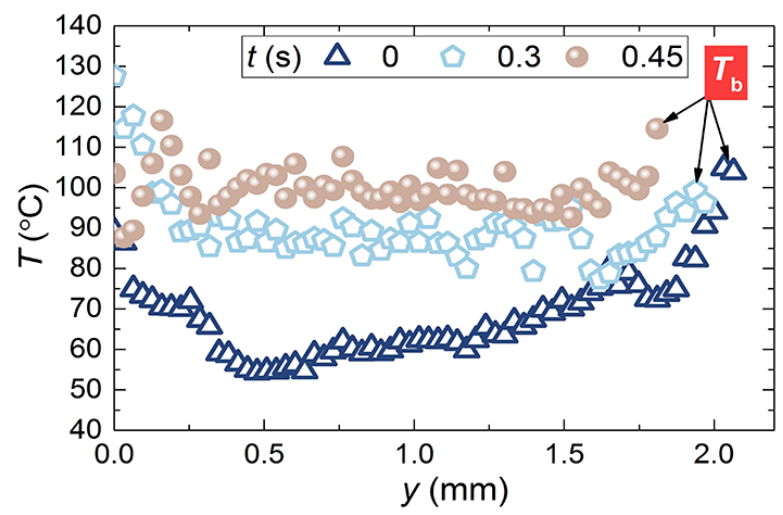

(a)

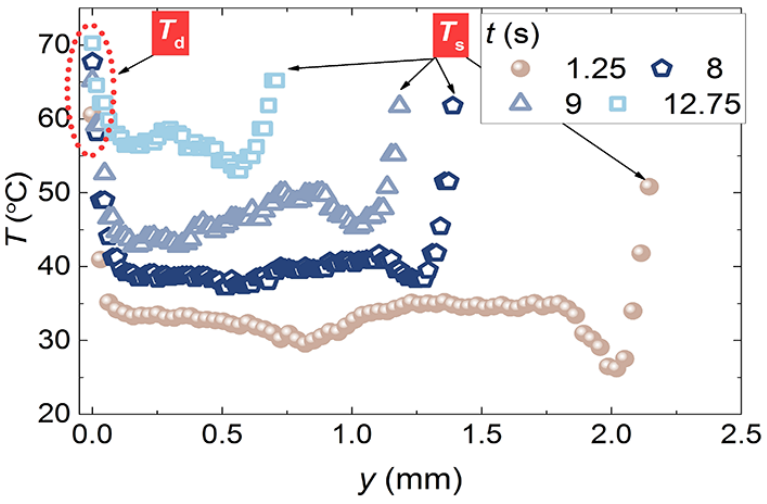

(b)

FIG. 5: Mean temperature profiles in heterogeneous droplet sections (OY section; see Fig. 2): (a) water/kerosene droplet $\left(V_{d} \approx\right.$ $\left.15 \mu \mathrm{l}, R_{d} \approx 1.53 \mathrm{~mm}, \gamma \approx 50 \mathrm{vol} . \%, U_{a} \approx 3 \mathrm{~m} / \mathrm{s}\right)$ at $T_{a} \approx 500^{\circ} \mathrm{C}$; (b) graphite suspension droplet $\left(V_{d} \approx 20 \mu \mathrm{l}\right.$ and $\delta \approx 2.3 \mathrm{~mm}$, $V_{\text {inc }} \approx 8 \mathrm{~mm}^{3}, U_{a} \approx 3 \mathrm{~m} / \mathrm{s}$ ) at $T_{a} \approx 500^{\circ} \mathrm{C}$; the standard deviation of $T$ is not more than $7 \%$ 


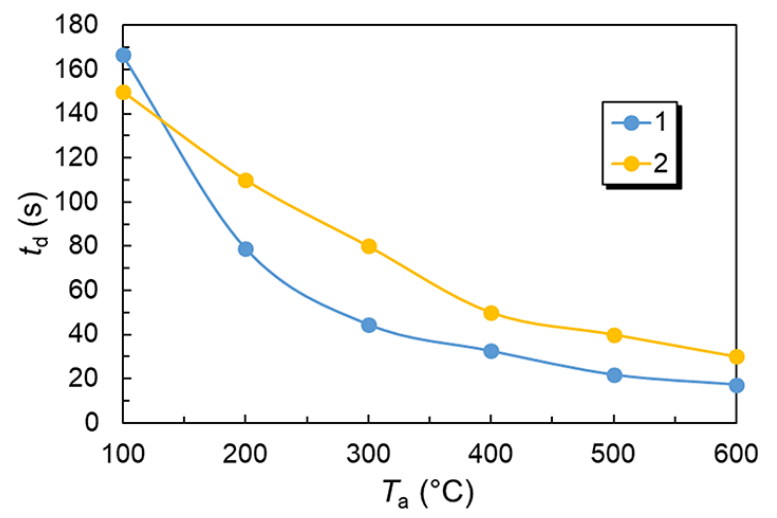

FIG. 6: Complete evaporation time of water in heterogeneous (with the internal particle) (1) and homogeneous (2) droplets: $1-V_{d} \approx 20 \mu \mathrm{l}, U_{a} \approx 3 \mathrm{~m} / \mathrm{s}$ (results of this study); $2-V_{d} \approx 20 \mu \mathrm{l}, U_{a} \approx 3 \mathrm{~m} / \mathrm{s}$ (based on data from Kuznetsov et al., 2018b)

Figure 4(b) demonstrates the temperature distribution in the OY section of a two-component water/kerosene droplet at different times from the beginning of the heating. Further, using temperature fields obtained, the temperature profiles were constructed in the OY section of a two-component droplet. Figure 5(a) presents the water temperatures in the OY section of the two-component droplet at $T_{a} \approx 500^{\circ} \mathrm{C}$. The water temperature levels out during the heating time. However, before the heterogeneous droplet disintegration, the water temperature in the central part of a droplet reaches or even exceeds $80-100^{\circ} \mathrm{C}$. Thus, the key condition for the two-component liquid droplet explosion is the water reaching the volumetric boiling point.

The experimental findings develop the concepts formed on the basis of data Kuznetsov et al. (2018a). Tests (Kuznetsov et al., 2018a) enabled finding of the noticeable temperature difference along the thickness of the liquid layer in the case when the graphite plate lies at the bottom of a reservoir. Based on the results of the study by Kuznetsov et al. (2018a), it is assumed that being heated by passing a thermal radiation through the liquid layer, this plate accelerates the warming-up of a liquid. The same conclusion is suitable for this research.

Note the originality of the physics of the investigated processes, the scientific and practical significance of the results obtained for understanding the latter. Fields and temperature distributions constructed on characteristic sections of two-component droplets showed that the mechanisms and criteria for micro-explosive fragmentation are quite close when considering solid inclusions and liquid admixture in water. As two typical criteria for the investigated processes of micro-explosive fragmentation of droplets, we can consider the following: achievement of temperature at the intercomponent boundary of values corresponding to boiling water $\left(80-100^{\circ} \mathrm{C}\right)$; the size of a drop with formed bubbles inside it exceeds 3-4 initial sizes of a drop. The temperature fields obtained by PLIF showed that for the fragmentation of droplets, it is not necessary to warm the entire intercomponent border to the boiling point.

In almost all experiments, local overheating regions were recorded. Their sizes were 8-12 times smaller than the drop size. In the case of a solid particle in a droplet, heating to micro-explosive boiling occurred longer than droplets containing a liquid combustible component. The registration of the fields of temperature and the nucleation of bubbles showed that many more bubbles are generated on the surface of a solid particle, but they are very small. At the intercomponent boundary between the two liquids, fewer bubbles appeared, but they grew in size much faster. This led to the disintegration of the two-fluid drop in a short time. Analysis of the results made it possible to single out a key feature, which was that in the case of water droplets with a liquid combustible component, the heating intensification is due to a larger group of factors than when considering a scheme with a drop containing a solid particle. In particular, a flammable liquid (for example, kerosene, oil, diesel, or others) has significantly lower vaporization heat and a markedly higher radiation absorption capacity compared to water. In addition, the viscosity and surface tension of the flammable liquid change significantly at heating. For water, the changes are not so significant. These factors contribute not only to the intensification of the drop heating due to three mechanisms (conduction, convection, radiation), but also lead to the weakening of bonds at the intercomponent boundary and the free surface of the drop. As a result, the droplet fragments with forming a cloud of small droplets. 
When considering the second criterion of micro-explosive fragmentation of droplets after reaching the critical sizes of bubbles inside the latter, (3-4) $R_{d}$ should be singled out as the general range. That is, if, as a result of filling with water vapor, the droplet increased in size by 3-4 times, then micro-explosive fragmentation necessarily occurred. The maximum increase in size recorded by the video camera before the droplet fragmentation reached (6-7) $R_{d}$. At the same heating temperatures and the volume of water in the composition of inhomogeneous droplets, significantly greater swelling was recorded in experiments with a liquid combustible component. Obviously, this is due to the synergistic effect, which consists of the fact that during heating the viscosity and surface tension of the liquid fuel and non-combustible components decreases, as well as the interfacial tension. In addition, the significant transformation of the surface of two-fluid droplets during heating leads to additional acceleration of thermogravitational convection. As a result, the drop becomes unstable and is intensively crushed.

The key novelty of the present study results also lies in the fact that reliable experimental information about the temperature in an inhomogeneous droplet has been obtained. Of course, the main interest is the temperature values near the intercomponent and free boundary of the drop. But, the temperature values in the whole drop volume are also important. This is because quite often when modeling, an assumption is made about uniform heating of liquid droplets and the expenditure of most of the supplied energy on phase transformations. The obtained temperature distributions and fields showed that even in homogeneous parts of the droplets (filled only with water or a liquid combustible component) there are significant temperature differences (see Figs. 4 and 5) during the whole heating time. These results are important for the development of modern models of evaporation and micro-fragmentation of droplets. Also important is the fact that both of the selected criteria for explosive fragmentation of droplets have been performed in all experiments when the droplet fragmentation was recorded.

Thus, when analyzing the recorded temperature distributions and fields, several key conclusions can be made. First, when conducting experiments and mathematical modeling, we can use either of two criteria to record the conditions of micro-explosive fragmentation of drops and warm-up times until these conditions are reached. Secondly, the temperature fields of the studied droplets are substantially non-uniform throughout the entire heating time. For micro-explosive fragmentation of such droplets, it is sufficient to achieve values corresponding to boiling water in local areas. The sizes of such areas can be $10 \times$ smaller than the initial drop size. In these areas, bubbles with water vapor originate, combine, grow in size, and lead to the destruction of a drop. Thirdly, the actual task of future research is to record the temperature in the liquid combustible component to demonstrate critical temperature difference sufficient for micro-explosive fragmentation of droplets. Obtaining reliable experimental data on this issue will allow us to determine the most effective heating schemes for two-fluid droplets for the purpose of micro-explosive fragmentation.

\subsection{Temperature Variations in Heterogeneous Droplets}

Figure 7(a) demonstrates how temperature variation $\left(\Delta T=T_{b}-T_{c}\right)$ in the two-component liquid droplet [Fig. 2(b)] occurs with heating time. Figure 7(a) has the shaded area, which denotes the $\Delta T$ values corresponding to the explosive disintegration and boiling of the two-component water/kerosene droplet. The explosive breakup of a two-component droplet occurs sustainably only at $T_{a}>200^{\circ} \mathrm{C}$. The heating rates at $T_{a}<200^{\circ} \mathrm{C}$ are very similar throughout the droplet. Such uniform heating results in the rapid liquid evaporation and boiling on the free surface. In the case when the temperature at the water/flammable liquid interface exceeds the temperature inside the droplet at $T_{a}>200^{\circ} \mathrm{C}$, the disintegration and boiling occur.

Figure 7(b) represents the transition from a heterogeneous temperature field to a homogeneous one $(\Delta T=$ $T_{d}-T_{s}$ ) inside water film of a graphite suspension droplet [Fig. 2(a)]. At the beginning of heating, the temperature at the inner interface of a suspension droplet $T_{d}$ is higher than the temperature of the free surface $T_{s}$. However, during the heating, the temperatures $T_{d}$ and $T_{s}$ level out.

\subsection{Explosive Breakup Regimes of Two-Component Liquid Droplets}

Using a high-speed video recording, we distinguished three explosive breakup regimes. The first regime is characterized by the droplets produced during the disintegration in size from 0.3 to $1.2 \mathrm{~mm}$, the heating time until the heterogeneous droplet breakup up to $6 \mathrm{~s}$, the concentration $\gamma=7-33 \mathrm{vol} . \%, T_{a}=200-215^{\circ} \mathrm{C}$, the $3-10$ large 


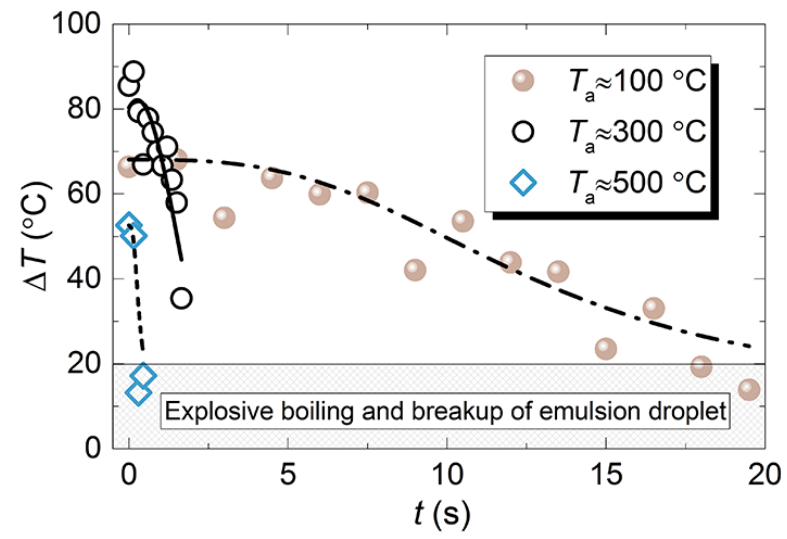

(a)

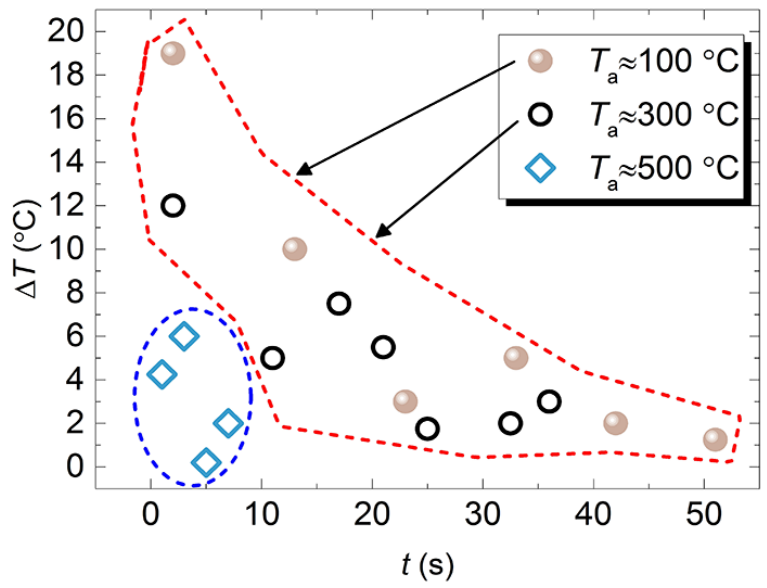

(b)

FIG. 7: Variation of the parameter $\Delta T$ in the course of heterogeneous droplet heating in the hot airflow at different $T_{a}$ : (a) twocomponent water/kerosene droplet ( $V_{d} \approx 15 \mu \mathrm{l}, R_{d} \approx 1.53 \mathrm{~mm}, \gamma \approx 15 \mathrm{vol} . \%, U_{a} \approx 3 \mathrm{~m} / \mathrm{s}$ ), (b) graphite suspension droplet $\left(V_{d} \approx 20 \mu \mathrm{l}, U_{a} \approx 3 \mathrm{~m} / \mathrm{s}\right)$

fragments formed after breakup, and the increase in total area of the evaporation surface by no more than $1.5-2.5 \times$. The second regime involves the medium size of the droplets produced after disintegration, up to $0.7 \mathrm{~mm}$, and the corresponding times of heterogeneous droplet destruction as well as the concentration $\gamma=33-66$ vol. \%, $T_{a}=280$ $300^{\circ} \mathrm{C}$, the heating time until the heterogeneous droplet breakup up to 4-6 s, the 10-30 liquid fragments formed, the total duration of the boiling and further breakup up to $0.8-1 \mathrm{~s}$, and the increase in total area of the evaporation surface by $3-4 \times$. The third one presents the minimum lifetimes, less than $3 \mathrm{~s}$, and size of the emerging droplets, less than $0.1 \mathrm{~mm}$. The number of the emerging droplets was maximum, from several dozens to one hundred. The concentration $\gamma$ should be higher than $66 \mathrm{vol} . \%$. The total area of the evaporation surface may increase by almost $8 \times$. The number of liquid fragments can reach 200-250. The temperature $T_{a}$ has to be higher than $400^{\circ} \mathrm{C}$. The heating time of a droplet until its destruction equals to $0.3-0.5 \mathrm{~s}$. Thus, we conclude that the dimensions of a droplet play a secondary role as compared to $T_{a}$ and $\gamma$ in identifying the explosive breakup regime.

The analysis of the videos of the experiments enables us to establish the important differences of the nucleation and growth mechanisms of vapor bubbles in the droplets containing the solid particle and in the droplets of two immiscible fluids. These differences are that for the two-component liquid droplets the strong heating results in emergence of a numerous group of the water micro-droplets near the boundary between the components. These micro-droplets often coalesce during heating and then fill with vapor. The bubble size increases with time. This results in filling the whole droplet in the vapor. When the entire droplet increments by $2-4 \times$ as compared to its initial size, the micro-explosion process of the two-component droplets occurs. In the droplet with the solid particle, the micro-droplets do not form. After warming-up the particle surface, the bubbles nucleate and then grow in size. After their 2-3-fold growth, they detach from the particle surface and coalesce. As in the tests with the two-component droplets, the micro-explosion of the droplet with the solid particle requires the droplet to be filled with vapor until its size will be $2-4 \times$ greater than the initial size. These critical values will be employed during the numerical simulation of the corresponding processes as criteria of the explosive breakup (Fu et al., 2002; Zeng and Lee, 2007; Shinjo et al., 2014; Zhang et al., 2018). In general, the criterion of the explosive breakup that involves the water heating up to the boiling point (Fu et al., 2002; Zeng and Lee, 2007; Shinjo et al., 2014; Zhang et al., 2018) takes place before another criterion. The latter lies in the increase of the droplet size by $2-4 \times$ relative to the initial one due to its filling with the vapor. If to generalize, in actual technologies both the criteria of the explosive breakup of the liquid droplets likely perform.

In addition, the performed experiments and data (Suzuki et al., 2011; Tarlet et al., 2016; Moussa et al., 2018) allowed the conclusion that to produce a fine aerosol through the explosive breakup of the liquid droplets it is necessary 
to provide not only the suitable heat fluxes but also the concentrations of components. The tests with the solid particle show that the thickness of the water film around the solid particle plays an important role. If the thickness is less than $0.1 \mathrm{~mm}$, it evaporates rapidly and does not overheat until micro-explosion. If the thickness is larger than $1 \mathrm{~mm}$ the warming-up time until micro-explosion is quite large. Only when it equals to $0.3-0.5 \mathrm{~mm}$, the breakup occurs. For the droplets of two immiscible fluids, the intensive fragmentation (breakup) results from the noticeable difference in the concentration of the components, e.g., at 90 vol. $\%$ of water and $10 \mathrm{vol}$. \% of the combustible component or vice versa. There are several reasons for this observation. To increase the concentration of the liquid combustible component, the specific heat of the entire droplet decreases, and its thermal diffusivity increases. Moreover, the heat quantity required for the endothermic phase transition reduces. When the water content in the droplet is 90 vol. \%, it rapidly warms up to the high temperature sufficient for the local boiling of water and the further breakup. These results illustrate the prospects for the development of the technologies based on the explosive breakup of the droplets containing not only water and a liquid combustible component but also solid particles. Thus, the minimum warming-up time of droplets until the explosive breakup can be reached at the maximum differences of the concentrations of the components.

\subsection{Comparison of Temperature Fields of Water Droplets and Water-Based Graphite Suspension Droplets}

Figure 8 shows average temperature distributions obtained by performing experiments with homogeneous droplets and graphite suspension droplets at the identical initial volumes of water. The average temperature distribution in a heterogeneous droplet at $T_{a} \geq 500^{\circ} \mathrm{C}$ is significantly different from that of a homogeneous droplet almost from the beginning of the heating. Close $T$ are observed for a short time only, about $1 \mathrm{~s}$. After this time interval, a considerable energy accumulation at the inner interface contributes to a significant change in the temperatures throughout the whole droplet. The $T$ in a heterogeneous droplet grows faster than in a homogeneous one (Fig. 8). Thus, a heterogeneous droplet significantly overheats, boils, and breaks up within a short time at high heating temperatures. At the same time, only $15-20 \%$ of a homogeneous droplet evaporates. The transition from a heterogeneous temperature field of a water droplet to a homogeneous one depends strongly on the buffer vapor layer formed around the droplet. For a heterogeneous droplet at high heating temperatures, there is no transition from a heterogeneous to a homogeneous field, i.e., a temperature field of a heterogeneous droplet is highly inhomogeneous during the whole heating time until the explosive disintegration.

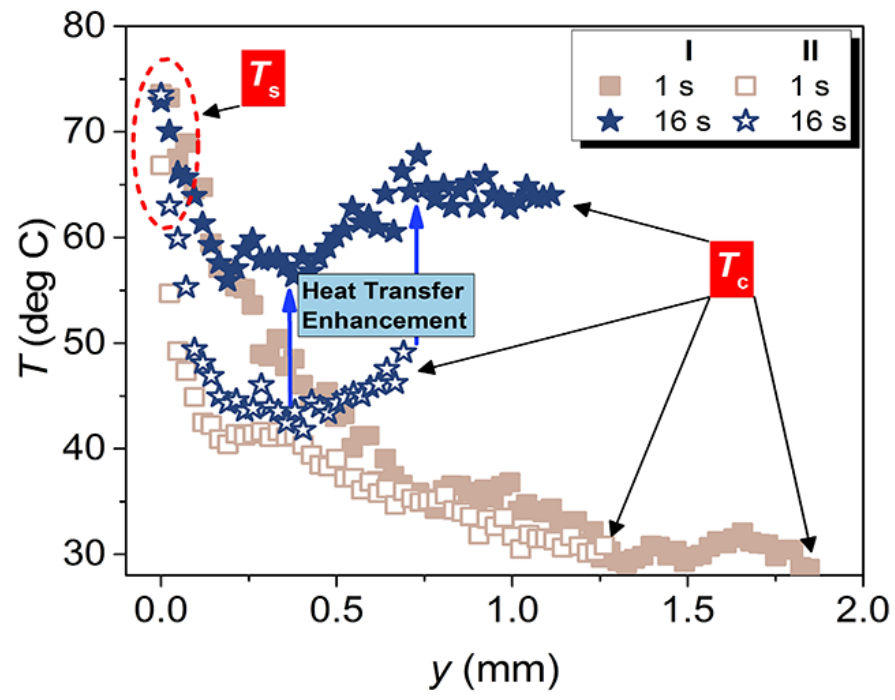

FIG. 8: Average temperature distributions in heterogeneous (I) and homogeneous (II) droplets of water at a heating temperature $T_{a}=500^{\circ} \mathrm{C}$; initial liquid volume of $20 \mu \mathrm{l}$ 
Thus, the research enables to substantiate the early-formulated assumption. It involves that solid particles and liquid combustible components in the droplets of water-based emulsions and suspensions can contribute to the enhancement of warming-up and the explosive breakup with the emergence of the aerosol cloud. The main mechanism involves that the heat quantity accumulated near the combustible component/non-combustible component boundary considerably increases. The larger the surface area of such boundaries, the stronger the explosive breakup, i.e., the more child droplets emerge.

The findings are of the great interest in the development of promising gas-vapor-droplet technologies because the intensive breakup helps to increase evaporation surface area. Consequently, it is possible to increase the warming-up and evaporation rates of droplets. In fuel technologies, this contributes to minimize the energy and time consumption during ignition, and to reduce an incomplete burning and anthropogenic emissions. In heat transfer technologies and during the thermal treatment of liquids, the breakup promotes evaporation enhancement in the small-sized heat chambers and under a low heat flux supplied. During firefighting, the explosive breakup results in covering a larger area of a heat source by the sprayed water. In addition, the breakup enhances all the main mechanisms of suppressing thermal decomposition and burning, namely, temperature decrease, substitution of oxidizer and pyrolysis products by the water vapor, accumulation of the heat energy of fire by a liquid due to its high specific heat, and evaporation heat.

\section{CONCLUSIONS}

1. Using the optical method PLIF, we recorded unsteady and highly inhomogeneous temperature fields of twocomponent liquid and suspension droplets when heated in a hot airflow. In addition, we established the main reasons for the rapid boiling and explosive breakup of heterogeneous droplets under intense heat.

2. Using PLIF for temperature measurements in suspension droplets, we demonstrated that the temperature variation in a droplet before boiling reaches $30-40^{\circ} \mathrm{C}$. Moreover, the temperature of the inclusion surface was higher than the temperature of the free liquid droplet surface.

3. The two-component liquid droplet breaks up in one of the three regimes. These regimes differ in duration and number of the emerging small droplets. The evaporation surface area of the liquid increases several-fold due to the explosive breakup of the two-component liquid droplets.

4. The particle influences the temperature distribution in a liquid phase of the suspension droplet considerably at the heating temperatures $T_{a}$ over $500^{\circ} \mathrm{C}$.

5. The main application of the research results involves obtaining specialized solutions to use as fluorophore when constructing the temperature fields and distributions of evaporating droplets of various fuel mixtures.

6. The experimental findings substantiate that the criterion based on heating of the non-combustible component, namely, water, up to the temperatures close to the boiling point $\left(80-100^{\circ} \mathrm{C}\right)$ is reasonable to use as the main one during the simulation of the explosive breakup of the droplets of suspensions and emulsions. Another criterion that lies in the increase of the droplet size by $2-4 \times$ relative to the initial one due to its filling with the vapor is also fulfilled. Its utilization is reasonable too. However, the heating up to the critical temperature is expedient to consider as a primary one.

\section{ACKNOWLEDGMENT}

The research was supported by the Russian Science Foundation (project 18-19-00056).

\section{REFERENCES}

Bochkareva, E.M., Miskiv, N.B., Nazarov, A.D., Terekhov, V.V., and Terekhov, V.I., Experimental Study of Evaporating Droplets Suspended Ethanol-Water Solution under Conditions of Forced Convection, Interf. Phenom. Heat Transf., vol. 6, no. 2, pp. 115-127, 2018.

Volume 6, Issue 4, 2018 
Charogiannis, A., An, J.S., and Markides, C.N., A Simultaneous Planar Laser-Induced Fluorescence, Particle Image Velocimetry and Particle Tracking Velocimetry Technique for the Investigation of Thin Liquid-Film Flows, Exp. Therm. Fluid Sci., vol. 68, pp. 516-536, 2015.

Fu, W.B., Hou, L.Y., Wang, L., and Ma, F.H., A Unified Model for the Micro-Explosion of Emulsified Droplets of Oil and Water, Fuel Process. Technol., vol. 79, pp. 107-119, 2002.

Gatapova, E.Ya., Kirichenko, E.O., Bai, B., and Kabov, O.A., Interaction of Impacting Water Drop with a Heated Surface and Breakup into Microdrops, Interf. Phenom. Heat Transf., vol. 6, no. 1, pp. 75-88, 2018.

Glushkov, D.O., Strizhak, P.A., and Chernetskii, M.Y., Organic Coal-Water Fuel: Problems and Advances (Review), Therm. Eng., vol. 63, no. 10, pp. 707-717, 2016.

Kuznetsov, G.V., Osipov, K.Y., Piskunov, M.V., and Volkov, R.S., Experimental Research of Radiative Heat Transfer in a Water Film, Int. J. Heat Mass Transf., vol. 117, pp. 1075-1082, 2018 a.

Kuznetsov, G.V., Piskunov, M.V., and Strizhak, P.A., Evaporation, Boiling and Explosive Breakup of Heterogeneous Droplet in a High-Temperature Gas, Int. J. Heat Mass Transf., vol. 92, pp. 360-369, 2016.

Kuznetsov, G.V., Piskunov, M.V., Volkov, R.S., and Strizhak, P.A., Unsteady Temperature Fields of Evaporating Water Droplets Exposed to Conductive, Convective and Radiative Heating, Appl. Therm. Eng., vol. 131, pp. 340-355, 2018 b.

Morozov, V.S., Volkov, R.S., and Misyura, S.Y., Visualizing the Velocity inside a Drop when a Cold Droplet Falls on a Sessile Drop on a Hot Wall, Interf. Phenom. Heat Transf., vol. 6, no. 3, pp. 209-218, 2018.

Moussa, O., Tarlet, D., Massoli, P., and Bellettre, J., Parametric Study of the Micro-Explosion Occurrence of W/O Emulsions, Int. J. Therm. Sci., vol. 133, pp. 90-97, 2018.

Nebuchinov, A.S., Lozhkin, Y.A., Bilsky, A.V., and Markovich, D.M., Combination of PIV and PLIF Methods to Study Convective Heat Transfer in an Impinging Jet, Exp. Therm. Fluid Sci., vol. 80, pp. 139-146, 2017.

Qubeissi, M.A. and Sazhin, S.S., Models for Droplet Heating and Evaporation: An Application to Biodiesel, Diesel and Gasoline Fuels, Int. J. Eng. Syst. Model. Simul., vol. 9, pp. 32-40, 2017.

Qubeissi, M.Al., Sazhin, S.S., and Elwardany, A.E., Modelling of Blended Diesel and Biodiesel Fuel Droplet Heating and Evaporation, Fuel, vol. 187, pp. 349-355, 2017.

Piskunov, M.V. and Strizhak, P.A., Using Planar Laser Induced Fluorescence to Explain the Mechanism of Heterogeneous Water Droplet Boiling and Explosive Breakup, Exp. Therm. Fluid Sci., vol. 91, pp. 103-116, 2018.

Sazhin, S.S., Modelling of Fuel Droplet Heating and Evaporation: Recent Results and Unsolved Problems, Fuel, vol. 196, pp. 69-101, 2017.

Shinjo, J., Xia, J., Ganippa, L.C., and Megaritis, A., Physics of Puffing and Microexplosion of Emulsion Fuel Droplets, Phys. Fluids, vol. 26, no. 10, p. 103302, 2014.

Strizhak, P.A., Piskunov, M.V., Volkov, R.S., and Legros, J.C., Evaporation, Boiling and Explosive Breakup of Oil-Water Emulsion Drops under Intense Radiant Heating, Chem. Eng. Res. Des., vol. 127, pp. 72-80, 2017.

Suzuki, Y., Harada, T., Watanabe, H., Shoji, M., Matsushita, Y., Aoki, H., and Miura, T., Visualization of Aggregation Process of Dispersed Water Droplets and the Effect of Aggregation on Secondary Atomization of Emulsified Fuel Droplets, Proc. Combust. Inst., vol. 33, pp. 2063-2070, 2011.

Tarlet, D., Josset, C., and Bellettre, J., Comparison between Unique and Coalesced Water Drops in Micro-Explosions Scanned by Differential Calorimetry, Int. J. Heat Mass Transf., vol. 95, pp. 689-692, 2016.

Volkov, R.S. and Strizhak, P.A., Planar Laser-Induced Fluorescence Diagnostics of Water Droplets Heating and Evaporation at High-Temperature, Appl. Therm. Eng., vol. 127, pp. 141-156, 2017.

Volkov, R.S., Kuznetsov, G.V., and Strizhak, P.A., Influence of Solid Inclusions in Liquid Drops Moving through a HighTemperature Gaseous Medium on Their Evaporation, Tech. Phys., vol. 59, pp. 1770-1774, 2014.

Vysokomornaya, O.V., Voytkov, I.S., Kuznetsov, G.V., and Abramova, A.V., High-Temperature Evaporation of Water Emulsion Droplets Used in Thermal Fluid Treatment, Int. J. Heat Mass Transf., vol. 126, pp. 1043-1048, 2018.

Zaitsev, A.S., Egorov, R.I., and Strizhak, P.A., Light-Induced Gasification of the Coal-Processing Waste: Possible Products and Regimes, Fuel, vol. 212, pp. 347-352, 2018a.

Zaitsev, D., Kirichenko, D., Shatekova, A., Ajaev, V., and Kabov, O.A., Experimental and Theoretical Studies of Ordered Arrays of Microdroplets Levitating over Liquid and Solid Surfaces, Interf. Phenom. Heat Transf., vol. 6, no. 3, pp. 219-230, 2018b.

Zeng, Y. and Lee, C.F., Modeling Droplet Breakup Processes under Micro-Explosion Conditions, Proc. Combust. Inst., vol. 31, 
pp. 2185-2193, 2007.

Zhang, Y., Huang, Y., Huang, R., Huang, S., Ma, Y., Xu, S., and Wang, Z., A New Puffing Model for a Droplet of ButanolHexadecane Blends, Appl. Therm. Eng., vol. 133, pp. 633-644, 2018.

Zubkov, V.S., Cossali, G.E., Tonini, S., Rybdylova, O., Crua, C., Heikal, M., and Sazhin, S.S., Mathematical Modelling of Heating and Evaporation of a Spheroidal Droplet, Int. J. Heat Mass Transf., vol. 108, pp. 2181-2190, 2017. 